\title{
Serum anti-GAPDH autoantibody levels reflect the severity of cervical lesions: A potential serum biomarker for cervical cancer screening
}

\author{
MEI LING XU ${ }^{1}$, HYOUNG JIN KIM ${ }^{1}$, SEUNG CHEOL KIM ${ }^{2}$, WOONG JU ${ }^{2}$, \\ YUN HWAN KIM ${ }^{2}$, KYU-HO CHANG ${ }^{3}$ and HONG-JIN KIM ${ }^{1}$ \\ ${ }^{1}$ Laboratory of Virology, College of Pharmacy, Chung-Ang University, Dongjak-Gu, Seoul 06974; \\ ${ }^{2}$ Department of Obstetrics and Gynecology, Ewha Woman's University College of Medicine, Yangcheon-Gu, \\ Seoul 03760; ${ }^{3}$ Department of Biological Engineering, Inha University, Incheon 22212, Republic of Korea
}

Received October 17, 2018; Accepted April 17, 2019

DOI: $10.3892 / \mathrm{ol} .2019 .10326$

\begin{abstract}
Recent studies have indicated that a certain level of autoantibodies may be essential for maintaining good health as well as preventing cancer development, and that the levels of serum autoantibodies can decline during malignant progression. The aim of the present study was to identify such an autoantibody-based biomarker for screening cervical lesions. An autoantigen reactive with healthy female sera was detected in the cytosolic fraction of HeLa cells, a cervical cancer cell line, and identified. Serum immunoglobulin (Ig)-G and IgM levels against the purified autoantigen in normal, cervical intraepithelial neoplasias (CINs) I, II and III, and cervical cancer were compared using ELISAs. The autoantigen in HeLa cells was identified to be GAPDH. The serum levels of anti-HeLa-GAPDH IgG decreased with increasing severity of cervical lesions, and similar decreases in IgM levels were revealed. Notably, the anti-HeLa-GAPDH IgG level was discovered to discriminate cervical cancer from normal samples with $80.0 \%$ sensitivity and $96.6 \%$ specificity. The serum anti-HeLa-GAPDH autoantibody level, as a single parameter, is a promising serum biomarker for screening cervical lesions.
\end{abstract}

\section{Introduction}

Cervical cancer is the third leading cause of cancer-associated mortalities in women in low-and middle-income countries (1). The 5-year survival rate of International Federation of Gynecology and Obstetrics (FIGO) stage I cervical cancer

Correspondence to: Professor Hong-Jin Kim, Laboratory of Virology, College of Pharmacy, Chung-Ang University, 84 Heukseok-Ro, Dongjak-Gu, Seoul 06974, Republic of Korea E-mail: hongjink@cau.ac.kr

Key words: autoantibody, glyceraldehyde 3-phosphate dehydrogenase, cervical cancer, cervical intraepithelial neoplasia, serum biomarker reaches $90 \%$ (2-4). However, $<16 \%$ of patients survive FIGO stage IV cancer $(4,5)$. Women who never participated in a cervical cancer screening program are associated with an incidence rate of cervical cancer $\geq 2$-fold higher than those previously screened (6). Therefore, increasing primary screening and early detection of cervical cancer are high priorities for improving survival rates.

The Papanicolaou (Pap) test is the most widely used test for primary screening of cervical cancer (7). However, accumulating data indicate that the test alone can miss cervical lesions due to its low sensitivity $(8,9)$. In certain developed countries, the human papillomavirus (HPV) DNA test is performed in combination with the Pap test to increase the accuracy of detection (10). The Pap test and the HPV DNA test require cells to be collected from the uterine cervix by a physician. This procedure can cause embarrassment and anxiety, and may lead to reluctance in being screened (11). In addition, cell-based tests are not suitable for high-throughput screening. Instead, serum offers advantages in the collection, storage and transportation of specimens. Therefore, serum-based strategies are considered advantageous for high-throughput screening.

Recent data suggest that $10-20 \%$ of cancer cases are associated with viral infections $(12,13)$. Therefore, serum antibodies acquired from virus infection are potential biomarkers for diagnosing cancer types, such as cervical cancer (14), nasopharyngeal carcinoma (15) and adult T-cell leukemia (16). HPV is detected in almost all patients with cervical cancer (17). Persistent HPV infection evokes humoral immune responses and there have been many attempts to use elevated levels of anti-HPV L1, E6 or E7 antibodies as markers of cervical lesions $(14,18)$. However, none of these biomarkers have proved to be sufficiently sensitive. Furthermore, $\geq 12$ types of HPV can be involved in the development of cervical cancer $(19,20)$ and there are technical barriers to preparing numerous different HPV antigens for assays. Therefore, discovering a serum biomarker for primary screening of cervical cancer remains a priority.

Autoantibodies serve important homeostatic roles by eliminating apoptotic and malignant cells and misfolded proteins (21-23), and changes in autoantibody levels are thought 
to occur in disease states (24-26). Autoantibody levels may be elevated in patients with autoimmune disease; elevated anti-nuclear antibody levels are revealed in $78 \%$ of patients with systemic lupus erythematosus and can be detected several years prior to disease onset (27). Elevated levels of autoantibodies have also been investigated in several types of cancer (28-30). However, these autoantibodies have drawbacks as independent cancer markers because they do not provide sufficient accuracy. Only $10-30 \%$ of patients with a given cancer have been demonstrated to be seropositive when single autoantibodies were used for screening $(31,32)$. Furthermore, not all autoantibodies exhibit an increase in patients with cancer (33-35).

Changes in protein expression and post-translational modifications occur during transitions to malignancy (36-39). In cancer cells, overexpression, unusual expression and misfolding of autoantigens, as well as aberrant post-translational modifications of autoantigens, can affect their recognition by the immune system in cancer (40), although the exact mechanism involved is unclear. Therefore, the use of cancer cell lines may be useful for identifying suitable autoantigens as cancer markers. HeLa cells, the most widely used cells in cancer research, have an altered pattern of protein expression when compared with normal cervical cell lines $(41,42)$. In the present study, HeLa cells were used as a source of autoantigens and for the first time a HeLa cell autoantibody was identified, whose level reflects the severity of cervical lesions.

\section{Materials and methods}

Specimens. The present study was carried out with the approval of the Institutional Review Board of the Ewha Woman's University Mokdong Hospital, Seoul, South Korea (approval no. EUMC 2016-07-067-002). The study was conducted in accordance with the Declaration of Helsinki. All serum samples were collected in a prospective and consecutive manner following the collection of written informed consent from the subjects.

A total of 130 serum samples were collected from female patients diagnosed with no cervical abnormalities $(n=29)$, cervical intraepithelial neoplasia (CIN) I $(n=18)$, CIN II $(n=23)$, CIN III $(n=30)$ and cervical cancer $(n=30)$, and stored at $-80^{\circ} \mathrm{C}$. All participants underwent a liquid-based cytology examination and those who tested positive for any abnormality in the cervix were designated for biopsy. Each cervical lesion was graded on the basis of hematoxylin and eosin staining of sections of formalin-fixed and paraffin-embedded tissue samples. Hysterectomy specimens with negative results in the hematoxylin and eosin staining examination were classified as normal. Cervical cancer was graded according to the FIGO staging system. All women $>20$ years old with abnormalities detected in the cervical cytology examinations, and who were designated for biopsy or surgery under suspicion of cervical CINs or cervical cancer, were included. Immunosuppressed individuals (those with human immunodeficiency virus infection, having undergone a transplant operation or on any immunosuppressive medication) or individuals with histories of other types of cancer, were excluded. The sera from the normal group were collected following the examination of the hysterectomy specimens. The sera from the CIN I group were collected immediately following punch biopsy, and those from the CIN II and CIN III groups were collected prior to large loop excision of transformation zones. The sera from the patients with cervical cancer were collected prior to surgery.

Extraction of cytosolic proteins from HeLa cells. The HeLa cell line, obtained from Korean Cell Line Bank, were maintained in Dulbecco's Modified Eagle's medium supplemented with $10 \%$ fetal bovine serum (InvivoGen, San Diego, CA, USA), $100 \mathrm{U} / \mathrm{ml}$ penicillin-streptomycin (GenDepot, Katy, TX, USA) and $25 \mu \mathrm{g} / \mathrm{ml}$ normocin (InvivoGen). The HeLa cells were detached from dishes with trypsin-EDTA (GenDepot), and cell pellets were collected by centrifugation $(400 \mathrm{x} g$ for 5 min). The pellets were washed once with PBS and recovered by centrifugation ( $400 \mathrm{x}$ g for $5 \mathrm{~min}$ ). To obtain the cytosolic proteins, the cell pellet was lysed with lysis buffer $(20 \mathrm{mM}$ Tris, $20 \mathrm{mM} \mathrm{NaCl}, 1 \mathrm{mM}$ EDTA, 5\% glycerol, $0.1 \%$ Triton $\mathrm{X}-100$ and $0.1 \% \beta$-mercaptoethanol; $\mathrm{pH} 5.7 ; 4^{\circ} \mathrm{C} ; 12 \mathrm{~h}$ ) and the cell debris was removed by centrifugation ( $400 \mathrm{x} \mathrm{g}$ for $5 \mathrm{~min}$ ).

Cation-exchange chromatography for the isolation of HeLa-GAPDH. The loading sample was prepared by adding 10 volumes of binding buffer $(20 \mathrm{mM}$ Tris, $20 \mathrm{mM} \mathrm{NaCl}$, $1 \mathrm{mM}$ EDTA, 5\% glycerol, $0.02 \%$ Triton $\mathrm{X}-100$ and $0.1 \%$ $\beta$-mercaptoethanol; $\mathrm{pH}$ 5.7) to the HeLa cell cytosolic proteins. A total of $1 \mathrm{ml}$ POROS XS resin (Thermo Fisher Scientific, Inc., Waltham, MA, USA) was equilibrated with binding buffer and the sample was loaded onto the resin. Unbound proteins were collected, the column was washed with 10 resin volumes of binding buffer, and the bound proteins were eluted by successive additions of elution buffer consisting of binding buffer containing 25-375 $\mathrm{mM} \mathrm{NaCl}$ at $25 \mathrm{mM}$ increments. The loading sample, flow-through, column wash and eluates were fractionated on a $12.5 \%$ polyacrylamide gel and the proteins were visualized by silver staining.

Western blotting. The proteins were fractionated by SDS-PAGE on $12.5 \%$ gels and transferred to polyvinylidene difluoride membranes (Merck KGaA, Darmstadt, Germany). Concentration of HeLa cytosolic proteins were determined by Bradford protein assay and 9 or $18 \mu \mathrm{g}$ of the proteins were loaded in each lane. Equal volume of samples eluted from cation-exchange chromatography were loaded to perform SDS-PAGE and Western blotting analysis. Mixtures of sera from the group of control females $(n=20)$ and rabbit anti-human GAPDH polyclonal antibody (cat. no.10094-T52, Sino Biological, Beijing, China) were diluted 1:200 in TBS containing 0.1\% Tween-20 (TBST) and $2.5 \%$ skimmed milk, and 1:1000 in TBST containing $0.5 \%$ skimmed milk, respectively, and reacted with the membranes for $2 \mathrm{~h}$ at room temperature. Following washing with TBS with $1 \%$ Tween-20, horseradish peroxidase (HRP)-conjugated goat anti-human IgG (Sigma-Aldrich; Merck KGaA) or HRP-conjugated goat anti-rabbit IgG (Bethyl Laboratories, Inc., Montgomery, TX, USA) was incubated with the membranes for $1 \mathrm{~h}$ at room temperature. The signals were developed using an AB Signal Chemiluminescence kit (AbClon, Inc., Seoul, South Korea) and photo activated on AGFA film (AGFA Healthcare, Ghent, Belgium). Rabbit anti-beta actin polyclonal antibody (cat. no.sc-1615-R, Santa Cruz Biotechnology, Texas, USA), diluted 1:1,000 in TBST containing $0.5 \%$ skimmed milk, was used as a positive internal loading control. 
Identification of the major protein purified by cation-exchange chromatography. A total of $1 \mu \mathrm{g}$ of the protein fraction extracted with $275 \mathrm{mM} \mathrm{NaCl}$ in the cation-exchange chromatography was fractionated on a polyacrylamide gel and visualized by Coomassie blue staining. The protein band of molecular mass $37 \mathrm{kDa}$ was cut out and treated with trypsin. The tryptic peptides were separated and analyzed by nano ACQUITY ultra performance liquid chromatography coupled directly to a LTQ-orbitrap-mass spectrometer (LC-MS/MS). The spectra from the LC-MS/MS were processed using the SEQUEST (Thermo Quest, San Jose, CA, USA) software and the peak list files generated were used to query the National Center for Biotechnology Information using the MASCOT search program (Matrix Science Ltd., London, UK, http://www.matrixscience.com/).

ELISAs for measuring serum antibody against HeLa cell whole cytosolic protein, purified HeLa-GAPDH or recombinant GAPDH in the normal, CIN I, CIN II, CIN III and cervical cancer groups. A 96-well microplate (SPL Life Sciences, Pocheon, South Korea) was coated overnight at $4^{\circ} \mathrm{C}$ with 2,000 , $1,000,500,250$ or $125 \mathrm{ng} /$ well HeLa cell cytosolic protein, $50 \mathrm{ng} /$ well purified HeLa-GAPDH or $50 \mathrm{ng} /$ well recombinant GAPDH (rGAPDH; Sino Biological, China), and blocked with 5\% skimmed milk in PBS containing 0.05\% Tween-20 (PBST). To measure the serum antibody against HeLa cell cytosolic protein, a mixture of normal group sera $(n=5)$ and a mixture of cervical cancer group sera $(n=5)$ were diluted 1:400 and incubated in the plate for $90 \mathrm{~min}$ at $37^{\circ} \mathrm{C}$. To measure the serum anti-HeLa-GAPDH antibody (Total, $\mathrm{n}=130$; normal, $\mathrm{n}=29$; CIN I, $\mathrm{n}=18$; CIN II, $\mathrm{n}=23$; CIN III, $\mathrm{n}=30$; and cervical cancer, $n=30$ ) or anti-rGAPDH antibody levels (Total, $n=129$; normal, n=29; CIN I, n=17; CIN II, n=23; CIN III, n=30; and cervical cancer, $n=30$ ), individual sera were diluted 1:100 and incubated in a plate coated with HeLa-GAPDH or rGAPDH. The serum samples were diluted with $1 \%$ skimmed milk in PBST. HRP-conjugated goat anti-human IgG polyclonal antibody or HRP-conjugated goat anti-human IgM polyclonal antibody (both Sigma-Aldrich; Merck KGaA) was diluted 1:5000 in $0.5 \%$ skim milk in PBST and incubated in the wells for $1 \mathrm{~h}$ at $37^{\circ} \mathrm{C}$. The reactions were developed with o-phenylenediamine (Sigma-Aldrich; Merck KGaA) and measured at $492 \mathrm{~nm}$ using a FlexStation 3 Multi-Mode microplate reader (Molecular Devices, LLC, Sunnyvale, CA, USA). The plates were washed 3 or 5 times with PBST between reactions.

To exclude interference by non-specific signals due to direct binding of the serum to the polyethylene plate, a mirror plate without coating antigen was prepared, and serum and secondary antibody were incubated as described above. The final optical density (OD) data were obtained using the following equation: OD from ELISA coated with antigen-OD from ELISA without antigen.

Statistical analysis. The difference in antibody levels was analyzed using the Kruskal-Wallis test with Dunn's multiple comparison test (GraphPad Prism version 5.01; GraphPad Software, Inc., La Jolla, CA, USA). The difference in ages between the groups was analyzed using analysis of variance with Bonferroni correction (ANOVA; GraphPad Software, Inc., La Jolla, CA, USA). $\mathrm{P}<0.05$ was considered to indicate statistically significant differences. Statistical power values (1- $\beta$ error) were calculated using the $G^{*}$ Power v.3.1 program. Receiver operating characteristic (ROC) curves were plotted, and areas under the curves (AUCs) were obtained, using GraphPad Prism version 5.01. The sensitivity, specificity, negative predictive value (NPV), positive predictive value (PPV) and accuracy of each assay were determined from the ROC curves. Optimal cut-off values were determined from Youden's index, which yields maximum values of sensitivity plus specificity based on the ROC curves. The following parameters were calculated: Sensitivity=number of true positives/(number of true positives + number of false negatives); specificity=number of true negatives/(number of true negatives + number of false positives); NPV=number of true negatives/(number of true negatives + number of false negatives); $\mathrm{PPV}=$ number of true positives/(number of true positives + number of false positives); and accuracy $=($ number of true positives + number of true negatives)/(number of true positives + number of true negatives + number of false positives + number of false negatives).

\section{Results}

Serum IgG against total HeLa cell cytosolic proteins in the normal and cervical cancer groups. ELISAs were performed to investigate the overall levels of antibodies against HeLa cell cytosolic proteins in the normal and cervical cancer group. As observed in Fig. 1, the IgG level was significantly lower in the cervical cancer group than the controls, and the level decreased as the amount of cytosolic protein used for coating decreased. This result indicates that the overall level of serum IgG against the whole cytosolic protein fraction in the cervical cancer group is lower than in the normal group.

Identification of a HeLa cell cytosolic protein with strong reactivity with sera from healthy females. Western blot analysis was performed to detect any major protein among the HeLa cell cytosolic proteins that reacted strongly with the sera from healthy females. A 37-kDa protein with strong reactivity was revealed (Fig. 2) and was identified by LC-MS/MS. The protein was termed HeLa-GAPDH for the purposes of the present study and was identified as GAPDH isoform-1.

Purification of HeLa-GAPDH by cation-exchange chromatography. Cation-exchange chromatography was used to purify HeLa-GAPDH from the total cytosolic proteins. The protein was isolated with satisfactory purity in the $275-\mathrm{mM} \mathrm{NaCl}$ elution fraction (Fig. 3A). Western blotting confirmed that the isolated fraction reacted with control sera from healthy females and with anti-human GAPDH antibody (Fig. 3B and C). The purified protein was demonstrated to give a linear response with human sera and anti-human GAPDH antibody in ELISAs (data not shown).

Serum anti-HeLa-GAPDH antibody levels in normal, CIN I, CIN II, CIN III and cervical cancer samples. Levels of serum anti-HeLa-GAPDH IgG and IgM were investigated in the normal, CIN (I, II and III) and cervical cancer groups by ELISA. The clinicopathological characteristics of each group are as follows: A total of 130 serum samples were used and 


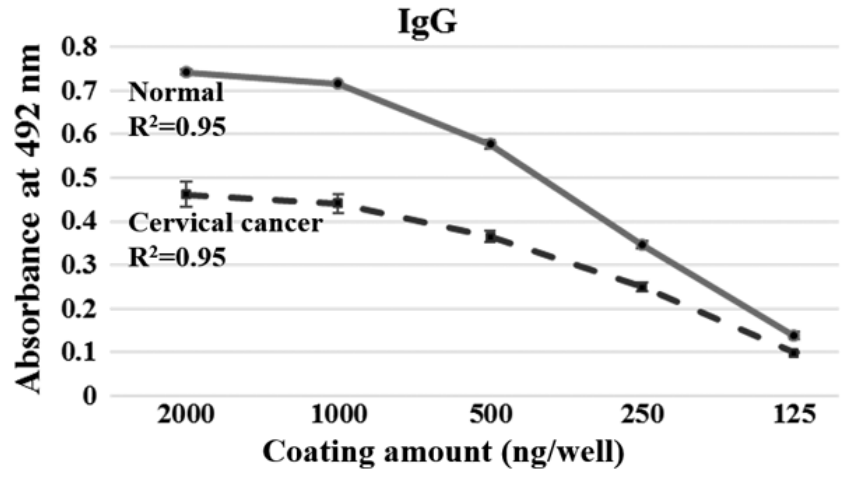

Figure 1. Linear response of serum IgG to total HeLa cell cytosolic protein fraction in ELISAs. The data are presented as the mean \pm standard deviation of two independent assays. $\mathrm{R}^{2}$ values were used to assess the linearity of the ELISAs. IgG, immunoglobulin G.

the average ages of the normal ( $n=29)$, CIN I $(n=18)$, CIN II $(\mathrm{n}=23)$, CIN III $(\mathrm{n}=30)$ and cervical cancer $(\mathrm{n}=30)$ groups were 43.6, 43.5, 45.7, 40.7 and 51.8 years, respectively. A significant difference of ages was observed between CIN III and cervical cancer groups (ANOVA with Bonferroni correction; $\mathrm{P}<0.05$ ). In the cervical cancer group, the proportions of squamous cell carcinoma, adenocarcinoma and adenosquamous carcinoma were $60.0,36.7$ and $3.3 \%$, respectively.

When compared with the anti-HeLa-GAPDH antibody levels in the normal group, the anti-HeLa-GAPDH IgG level of the CINs and cervical cancer groups generally declined as the severity of the cervical lesions increased (CIN II, $\mathrm{P}<0.01$ versus normal; CIN III, $\mathrm{P}<0.001$ versus normal; and cervical cancer, $\mathrm{P}<0.001$ versus normal; Fig. 4A). Similarly, anti-HeLa-GAPDH IgM was lower in the CIN and cervical cancer samples than in the controls (CIN II, $\mathrm{P}<0.05$ versus normal; and cervical cancer, $\mathrm{P}<0.001$ versus normal; Fig. 4B). When rGAPDH was used as the coating antigen for the ELISAs, reduced number of serum sample (Total, $n=129$; normal, $n=29$; CIN I, $n=17$; CIN II, $n=23$; CIN III, $n=30$; and cervical cancer, $n=30$ ) was used in measuring anti-rGAPDH antibody levels. However, the trends in levels of anti-rGAPDH IgG and anti-rGAPDH IgM in the groups were similar to when HeLa-GAPDH was used (Fig. 5).

The statistical power (1- $\beta$ ) and diagnostic performances (sensitivity, specificity, NPV, PPV, accuracy and AUC) were evaluated for the ability to discriminate the various cervical lesions from normal samples (Table I). It was demonstrated that the anti-HeLa-GAPDH IgG levels discriminated cervical cancer from healthy samples with high sensitivity $(80.0 \%)$ and specificity (96.6\%), whereas the anti-HeLa-GAPDH IgM levels discriminated cervical cancer from normal with $73.3 \%$ sensitivity and $72.4 \%$ specificity. Overall, higher AUCs for discriminating cervical cancer from normal were obtained with anti-HeLa-GAPDH IgG than with anti-HeLa-GAPDH IgM (AUC, 0.91 vs. 0.78, respectively; Fig. 6 and Table I). Therefore, anti-HeLa-GAPDH IgG may be a more reliable biomarker for detecting cervical cancer than anti-HeLa-GAPDH IgM.

CIN II is a clinically important end-point because CIN II and III can progress to cervical cancer if left untreated (43). Anti-HeLa-GAPDH IgM was revealed to discriminate CIN $\mathrm{II}^{+}$lesions (CIN II, CIN III and cervical cancer) from the normal plus CIN I group with $74.7 \%$ sensitivity and $53.2 \%$

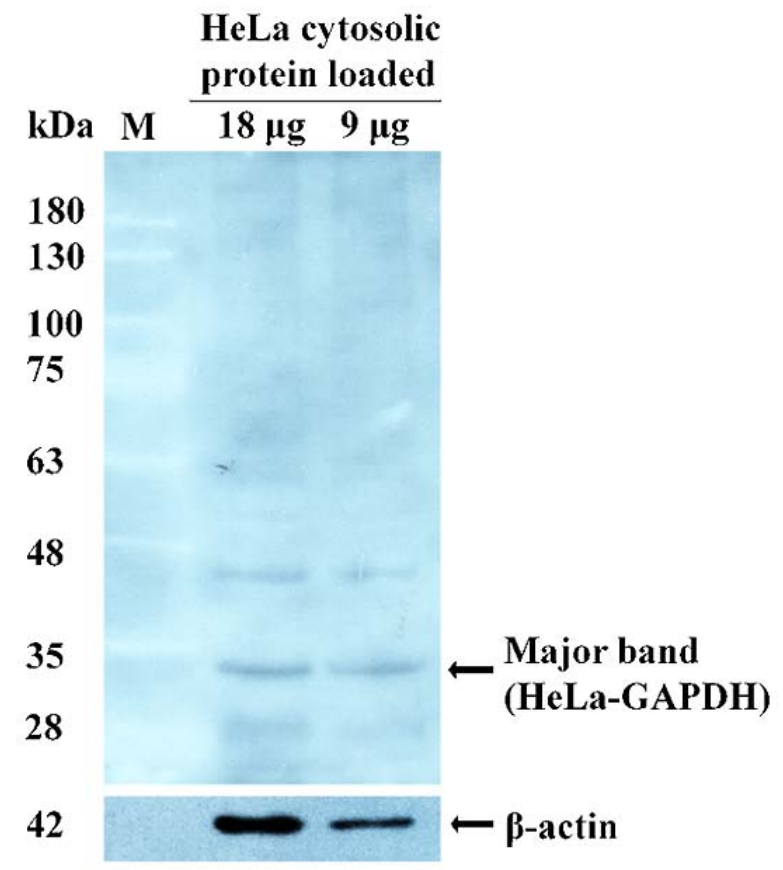

Figure 2. Reactivity of sera from healthy females against HeLa cell cytosolic proteins using western blotting. The arrow indicates a major HeLa cell cytosolic protein detected by the sera from healthy females. The major protein was identified as GAPDH isoform-1 using nano ACQUITY ultra performance liquid chromatography coupled directly to a linear trap quadrupole-orbitrap-mass spectrometer. $\beta$-actin polyclonal antibody was used as a positive internal loading control in western blotting.

specificity, and higher sensitivity (74.7\%) and specificity (72.3\%) were obtained with anti-HeLa-GAPDH IgG (Table I; normal + CIN I vs. CIN $\mathrm{II}^{+}$). In addition, the overall AUC values for anti-HeLa-GAPDH $\operatorname{IgG}$ were higher than those for anti-HeLa-GAPDH IgM for discriminating $\mathrm{CIN} \mathrm{II}^{+}$from the normal plus CIN I group (Table I; normal + CIN I vs. $\mathrm{CIN} \mathrm{II}^{+}$). Therefore, the results indicate that anti-GAPDH $\mathrm{IgG}$ reflects the severity of cervical lesions more accurately than anti-GAPDH IgM. It can be concluded that anti-GAPDH autoantibody is a potential serum biomarker for high-grade CINs and cervical cancer.

\section{Discussion}

High-grade CINs (II and III) have a high probability of progressing to cervical cancer (44-46). Therefore, discriminating CIN II and more severe cervical lesions from normal sample is important in primary screening for cervical lesions. The present study aimed to discover a new autoantibody-based marker for detecting cervical lesions and revealed that serum anti-GAPDH IgG or IgM may be a useful marker for that purpose. Anti-GAPDH IgG was identified to decrease with increasing severity of cervical lesions, and cervical cancer was detected with high sensitivity $(80.0 \%)$ and specificity (96.6\%) with anti-HeLa-GAPDH IgG as marker. In addition, a lower anti-HeLa-GAPDH IgG level discriminated CIN $\mathrm{II}^{+}$from normal and CIN I samples with $74.7 \%$ sensitivity and $72.3 \%$ specificity. The present findings suggest that a decreased anti-GAPDH IgG level is a promising biomarker for cervical cancer. 
A

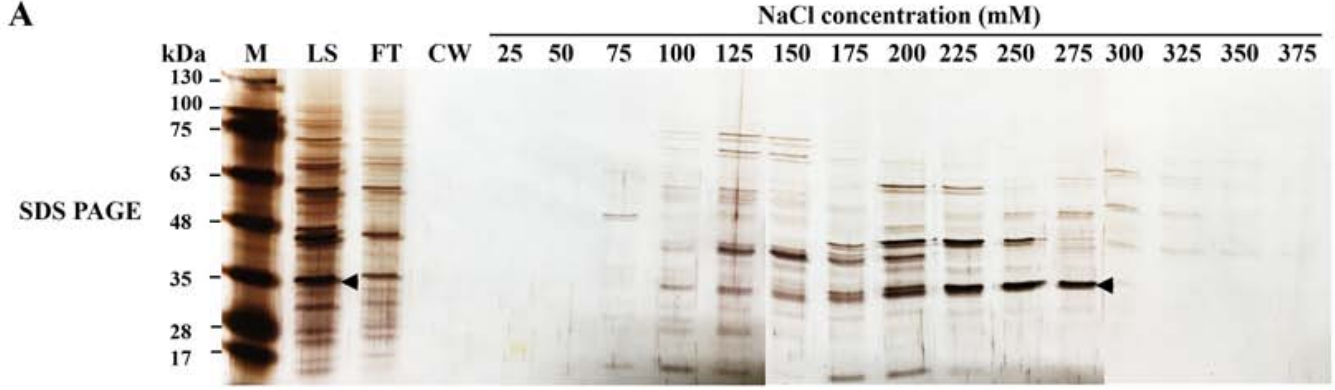

B

$\mathrm{NaCl}$ concentration $(\mathrm{mM})$

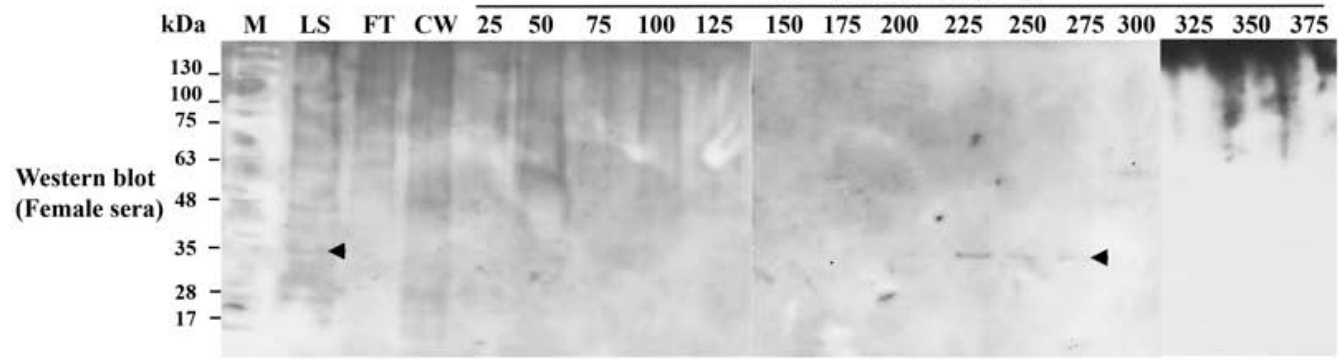

C

$\mathrm{NaCl}$ concentration $(\mathrm{mM})$

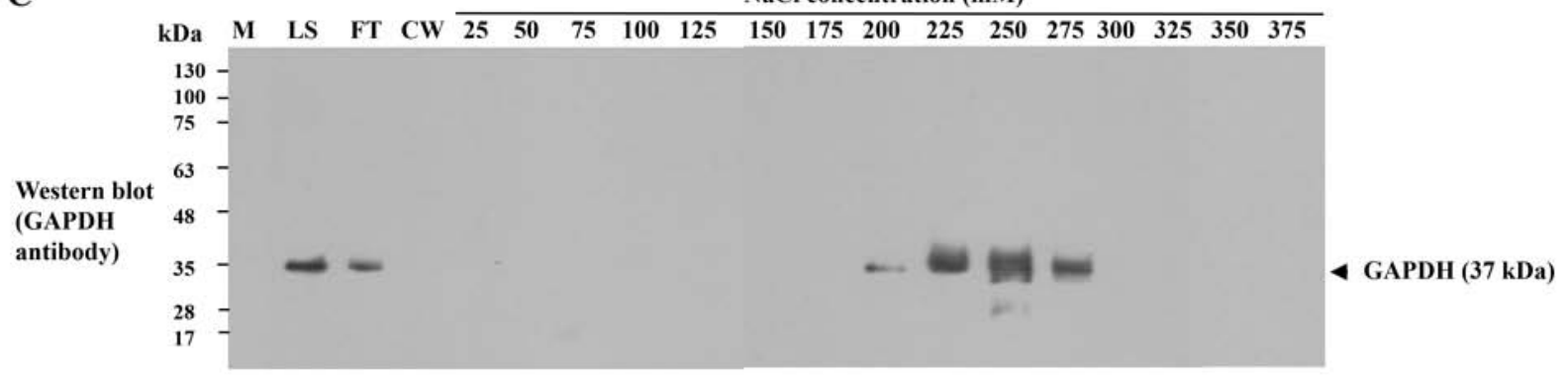

Figure 3. Purification of HeLa-GAPDH from HeLa cell cytosolic proteins by cation-exchange chromatography. (A) HeLa cell cytosolic proteins were separated by cation-exchange chromartography. The proteins were separated by SDS-PAGE and visualized with silver staining. (B) Western blot analysis of proteins from cation-exchange chromatography using healthy donor sera. (C) Western blot analysis where HeLa-GAPDH was detected in fractions from cation-exchange chromatography using anti-human GAPDH polyclonal antibody. HeLa-GAPDH is indicated by arrowheads. M, protein markers; LS, loading sample; FT, flow-through; CW, column wash.

Autoantibodies have received much attention as serum biomarkers for cancer screening over the past five decades (24,47-49). Misfolding, overexpression and aberrant glycosylation of autoantigens are recognized to be general features of cancer development $(36,38,39,50)$, and it was considered likely that alterations in the properties of autoantigens that can elicit humoral immune responses would be encountered (40). Therefore, previous attempts to discover autoantibody-based cancer markers have focused on autoantibodies whose levels increase in the presence of cancer. However, recent studies have demonstrated that an increase in the level of an autoantibody is only one of a variety of changes associated with autoantibodies in cancer. Autoantibodies not only eliminate the breakdown products of antigens resulting from apoptosis but also protect healthy cells from attack by the immune system of the individual $(21,23,51,52)$. In addition, autoantibodies can serve anti-oncogenic roles by promoting the apoptosis of malignant cells and reducing their invasiveness $(53,54)$, as well as by maintaining homeostasis $(55)$. Decreased levels of anti-glucose-regulated protein 78 and anti- $\alpha$-enolase 1 antibodies have been demonstrated in ovarian, breast and lung cancer $(34,35,53)$, and decreased levels of anti- $\alpha$-enolase 1 were revealed in late stage (stage IV) lung cancer but not in earlier stages (stage I/II) (34). The present observations on anti-GAPDH autoantibody levels during cervical cancer development together with these other findings suggest that maintaining a certain level of various autoantibodies is critical for the balance of the immune system, and that decreased levels can be associated with a malignant state.

GAPDH is a glycolytic enzyme that promotes the 6th step of glycolysis (56). Increased levels of GAPDH mRNA are detected in cervical cancer tissues and cell lines $(57,58)$. Malignant cells preferentially employ aerobic glycolysis rather than oxidative phosphorylation to generate adenosine triphosphate (59), and overexpression of glycolytic enzymes has been suggested to be a hallmark of cancer cell metabolism (60). Furthermore, GAPDH is associated with an increased migratory behavior and proliferation of cancer cells (61-63), and its downregulation by GAPDH segregator, a triazine-based small molecule, decreases the viability of colon carcinoma cells (64). In addition, treatment with antisense oligonucleotides against GAPDH, or glycolysis-targeting anticancer agents, including 3-bromopyruvate, was demonstrated to prevent the proliferation of human colon cancer cells and to induce apoptosis of human and mouse cervical carcinoma cells $(65,66)$. Therefore, it appears that GAPDH has anti-apoptotic or pro-proliferative 

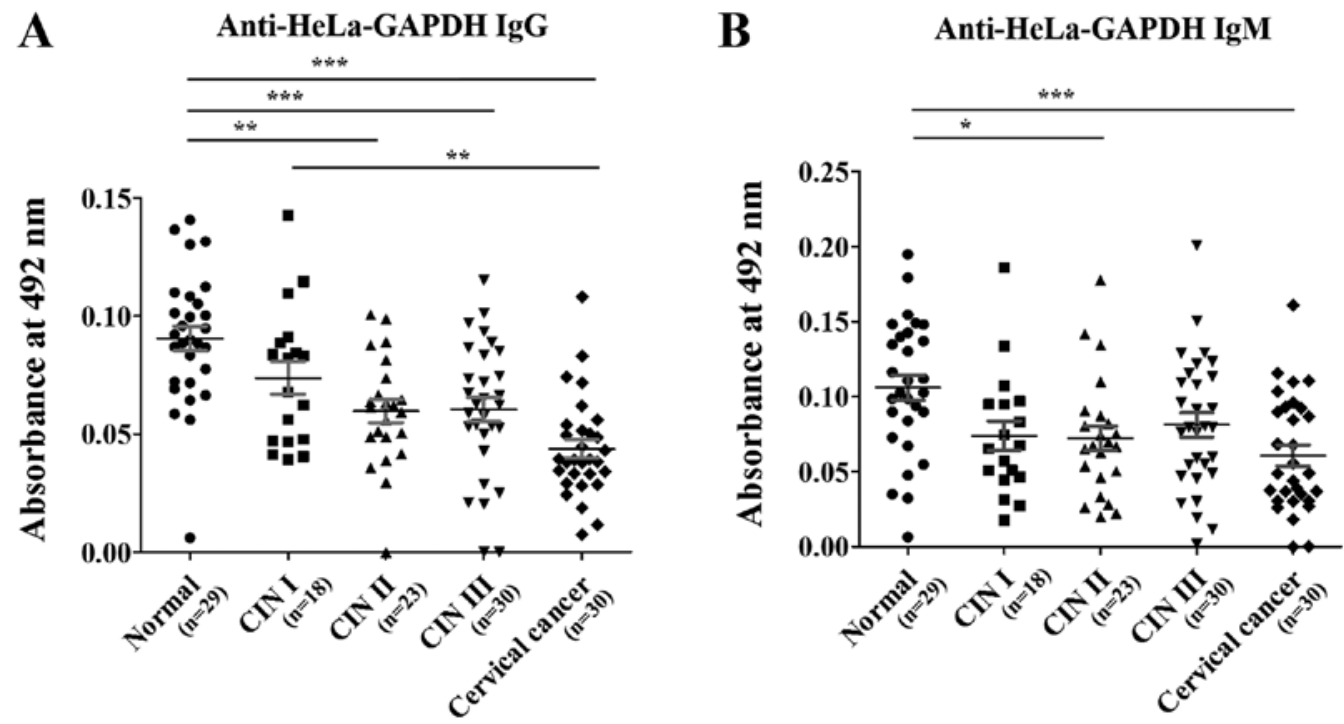

Figure 4. Anti-HeLa-GAPDH antibody levels in the normal, CIN I, CIN II, CIN III and cervical cancer groups. (A) Levels of anti-HeLa-GAPDH IgG. (B) Levels of anti-HeLa-GAPDH IgM. The central lines represent the mean values and the error bars indicate the ranges of the standard error of the mean. Total, $\mathrm{n}=130$; normal, $\mathrm{n}=29$; CIN I, n=18; CIN II, n=23; CIN III, n=30; and cervical cancer, $\mathrm{n}=30$. Statistical analysis was performed using the Kruskal-Wallis test with Dunn's multiple comparison test. ${ }^{*} \mathrm{P}<0.05,{ }^{* *} \mathrm{P}<0.01$ and $^{* * * *} \mathrm{P}<0.001$, as indicated. CIN, cervical intraepithelial neoplasia; IgG/IgM, immunoglobulin G/M.
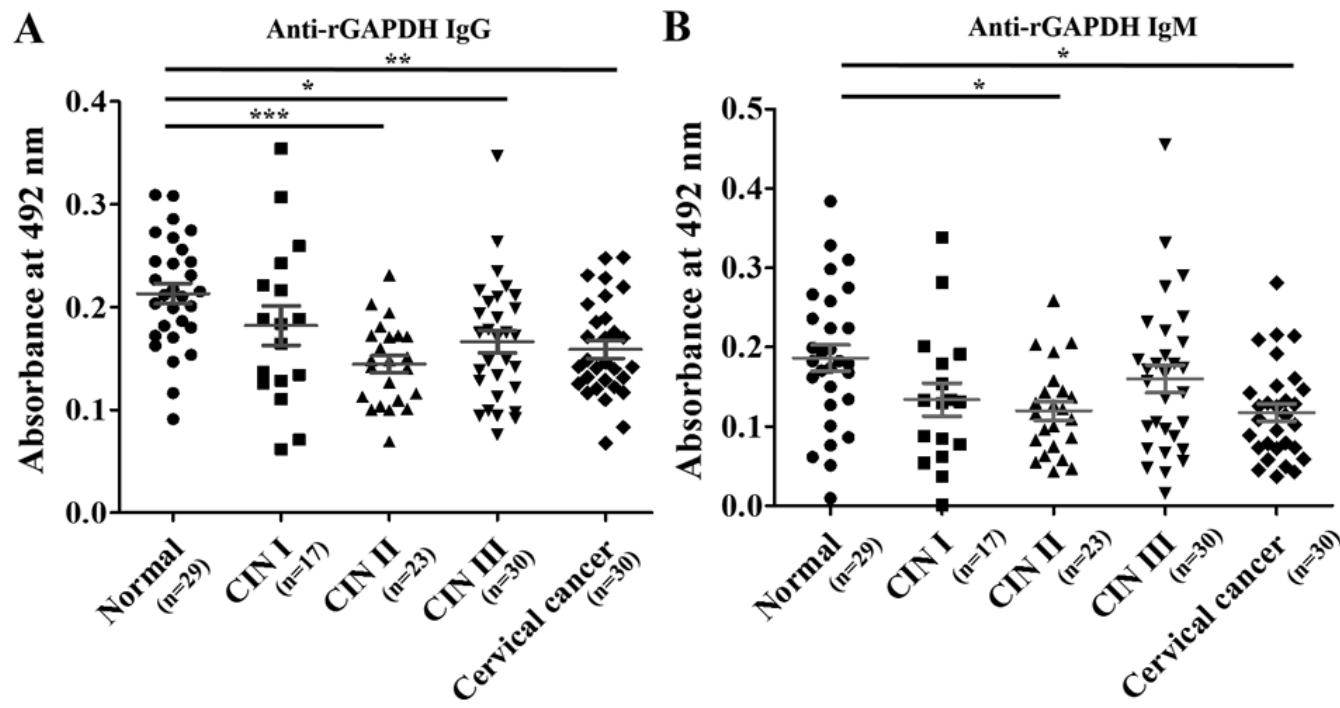

Figure 5. Comparison of anti-rGAPDH IgG and anti-rGAPDH IgM levels in the normal, CIN I, CIN II, CIN III and cervical cancer groups in ELISAs using rGAPDH as the coating antigen. (A) Levels of anti-rGAPDH IgG. (B) Levels of anti-rGAPDH IgM. The central lines represent the mean values, and the error bars indicate the ranges of the standard error of the mean. Total, $\mathrm{n}=129$; normal, $\mathrm{n}=29$; CIN I, $\mathrm{n}=17$; CIN II, $\mathrm{n}=23$; CIN III, $\mathrm{n}=30$; and cervical cancer, $\mathrm{n}=30$. Statistical analysis was performed using the Kruskal-Wallis test with Dunn's multiple comparison test. ${ }^{*} \mathrm{P}<0.05,{ }^{* *} \mathrm{P}<0.01$ and ${ }^{* * *} \mathrm{P}<0.001$, as indicated. rGAPDH, recombinant GAPDH; CIN, cervical intraepithelial neoplasia; IgG/IgM, immunoglobulin G/M.

activity for malignant cells, and that maintaining GAPDH levels may be critical for preventing malignancy. In light of the results of the present study and previous findings, it may be that a decrease in anti-GAPDH autoantibody levels in cervical cancer is a consequence of providing a favorable microenvironment for the survival and proliferation of cancer cells.

Panels of autoantibodies, generally ones that are elevated in cancer, have been used ascancer biomarkers toovercome theinsufficient sensitivity or specificity of single autoantibodies $(29,30,32)$. The EarlyCDT ${ }^{\circledR}$-Lung test, which uses a combination of 6 autoantibodies (anti-cellular tumor antigen p53, autoimmunogenic cancer/testis antigen NY-ESO-1, cancer-associated gene protein,
GBU4-5, Annexin I and transcription factor SOX2), provides $39 \%$ sensitivity and $89 \%$ specificity for identifying individuals at high risk of lung cancer (67). Similarly, the present research group had demonstrated that a combination of 3 autoantibodies [cancer antigen (CA)15-3, carcinoembryonic antigen and CA19-9 autoantibodies] discriminate cervical cancer from normal samples with $90.3 \%$ sensitivity and $82.1 \%$ specificity (68). In contrast with previous findings, a decrease in the level of a single autoantibody (IgG against GAPDH) was identified in the present study to discriminate cervical cancer from normal samples with $80.0 \%$ sensitivity and $96.6 \%$ specificity. This finding indicates that the reduced $\operatorname{IgG}$ response against the autoantigen may be useful as a biomarker. 
Table I. Statistical parameters based on anti-HeLa-GAPDH IgG or IgM levels for discriminating CINs or cervical cancer from normal cytology, and CIN II ${ }^{+}$from Normal + CIN I.

A, IgG

Comparison

Power (1- $\beta$ error) $)^{\mathrm{a}} \quad$ Sensitivity $^{\mathrm{b}}, \% \quad$ Specificity $^{\mathrm{c}}, \% \quad$ NPV, \% PPV, \% Accuracy, \% AUC

\begin{tabular}{|c|c|c|c|c|c|c|c|}
\hline Normal vs. CIN I & 0.48 & 72.2 & 65.5 & 79.2 & 56.5 & 68.1 & 0.69 \\
\hline Normal vs. CIN II & 0.99 & 73.9 & 82.8 & 80.0 & 77.3 & 78.8 & 0.83 \\
\hline Normal vs. CIN III & 0.98 & 83.3 & 65.5 & 79.2 & 71.4 & 74.6 & 0.79 \\
\hline Normal vs. Cervical cancer & 1.00 & 80.0 & 96.6 & 82.4 & 96.0 & 88.1 & 0.91 \\
\hline Normal + CIN Id vs. CIN II ${ }^{+e}$ & 1.00 & 74.7 & 72.3 & 61.8 & 82.7 & 73.8 & 0.78 \\
\hline
\end{tabular}

B, IgM

Comparison

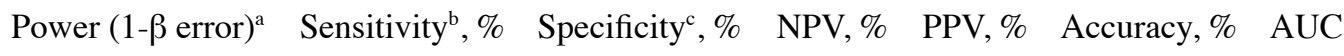

Normal vs. CIN I

0.68

0.80

0.55

0.98

0.80

Normal vs. Cervical cancer

Normal + CIN I ${ }^{\mathrm{d}}$ vs. CIN II ${ }^{+e}$

83.3
78.3
56.7
73.3
74.7

83.3

56.7

74.7

\section{1 \\ 72.4 \\ 75.9 \\ 72.4}

53.2
85.7
80.8
62.9
72.4
73.8

57.7

69.2

70.8

73.3

54.3

7.7
69.2
0.8
73.3
54.3

$\begin{array}{ll}70.2 & 0.73 \\ 75.0 & 0.74 \\ 66.1 & 0.67 \\ 72.9 & 0.78 \\ 66.9 & 0.65\end{array}$

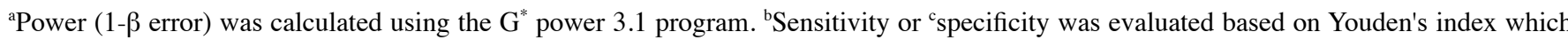
yields maximum values of sensitivity plus specificity. ${ }^{\mathrm{d}}$ Normal $+\mathrm{CIN}$ I: Normal and CIN I. ${ }^{\mathrm{e}} \mathrm{CIN}$ II ${ }^{+}$: CIN II, CIN III and cervical cancer. $\mathrm{NPV}$, negative predictive value; PPV, positive predictive value; AUC, area under the curve; CIN, cervical intraepithelial neoplasia; IgG/IgM, immunoglobulin G/M.
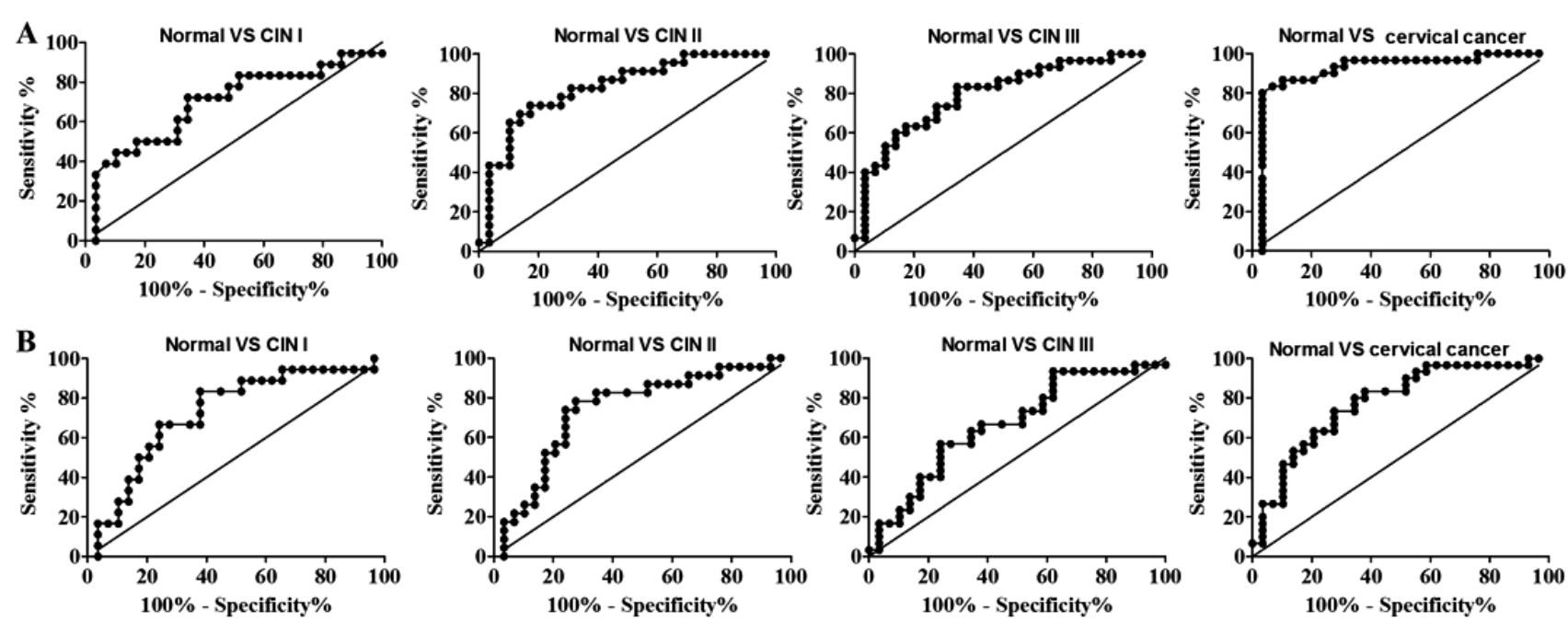

Figure 6. ROC curves for discriminating CIN I, CIN II, CIN III and cervical cancer from healthy specimens. (A) ROC curves for discriminating cervical lesions from normal cytology based on the anti-HeLa-GAPDH IgG levels observed in Fig. 4A. (B) ROC curves for discriminating cervical lesions from normal cytology based on the anti-HeLa-GAPDH IgM level observed in Fig. 4B. ROC, receiver operating characteristic; CIN, cervical intraepithelial neoplasia.

Overexpression of the GAPDH gene has been observed in breast, ovarian, colon, lung, liver and cervical cancer $(60,69-71)$. In addition, several types of non-cancerous cells, such as inflammatory cells, naïve T lymphocytes and endothelial cells also undergo rapid proliferation and obtain energy by aerobic glycolysis during their activation or proliferation which are involved in GAPDH gene expression (72). Therefore, it will be of the interest to examine the distinctive role of GAPDH in such non-cancerous cells as well as anti-GAPDH antibody levels in the non-cancerous status. Understanding the functions of anti-GAPDH autoantibody in cancer may provide new insights for the treatment of cancer.

The limitations of this study include a limited number of serum samples $(\mathrm{n}=130)$ used, although the resulting power (0.8-1.0) satisfied the criterion for reliability. In addition, the anti-GAPDH antibody levels were only investigated in a single ethnic group (Korean women). Therefore, further studies 
involving larger sample sizes and diversity are required to confirm whether the decreased anti-GAPDH level in patients with cervical lesions is a general feature.

In conclusion, the present study presents the first evidence that decreased levels of anti-GAPDH autoantibody (IgG and $\operatorname{IgM})$, particularly anti-GAPDH $\operatorname{IgG}$, is a potential serum biomarker for detecting CINs and cervical cancer. Furthermore, it was revealed that decreased levels of serum anti-GAPDH autoantibodies reflect the severity of cervical lesions. Serum anti-GAPDH IgG level, as a single indicator, was able to discriminate $\mathrm{CIN} \mathrm{II}^{+}$from normal plus CIN I with high sensitivity and specificity. It is expected that the present findings can provide a new paradigm to the discovery of serum biomarker for cervical lesions screening.

\section{Acknowledgements}

Not applicable.

\section{Funding}

The present study was supported by the Basic Science Research Program through the National Research Foundation of Korea (NRF) funded by the Ministry of Education (grant no. NRF-2015R1D1A1A01057370).

\section{Availability of data and materials}

The datasets used and/or analyzed during the present study are available from the corresponding author on reasonable request.

\section{Authors' contributions}

SCK, WJ and YHK collected the serum samples and cervical biopsies, and graded the cervical lesions. MLX, HyJK and HoJK conceived and designed the experiments. MLX and KC performed the experiments. MLX, HyJK, KC and HoJK analyzed the data. MLX and HyJK wrote the paper. HoK is responsible for the integrity of the work as a whole. All the authors have accepted responsibility for the entire consent of this submitted article and approved submission.

\section{Ethics approval and consent to participate}

The present study was carried out with the approval of the Institutional Review Board of the Ewha Woman's University Mokdong Hospital, Seoul, South Korea (approval no. EUMC 2016-07-067-002). The study was conducted in accordance with the Declaration of Helsinki. All serum samples were collected in a prospective and consecutive manner following the collection of written informed consent from the subjects.

\section{Patient consent for publication}

Not applicable.

\section{Competing interests}

The authors declare that they have no competing interests.

\section{References}

1. Torre LA, Siegel RL, Ward EM and Jemal A: Global cancer incidence and mortality rates and trends-an update. Cancer Epidemiol Biomarkers Prev 25: 16-27, 2016.

2. Kim SM, Choi HS and Byun JS: Overall 5-year survival rate and prognostic factors in patients with stage IB and IIA cervical cancer treated by radical hysterectomy and pelvic lymph node dissection. Int J Gynecol Cancer 10: 305-312, 2000.

3. Kasamatsu T, Onda T, Sawada M, Kato T, Ikeda S, Sasajima Y and Tsuda H: Radical hysterectomy for FIGO stage I-IIB adenocarcinoma of the uterine cervix. Br J Cancer 100: 1400-1405, 2009.

4. Survival rates for cervical cancer by stage.https://www.cancer. org/cancer/cervical-cancer/detection-diagnosis-staging/survival. html.

5. Bulk S, Visser O, Rozendaal L, Verheijen RH and Meijer CJ: Incidence and survival rate of women with cervical cancer in the Greater Amsterdam area. Br J Cancer 89: 834-839, 2003.

6. Ibáñez R, Alejo M, Combalia N, Tarroch X, Autonell J, Codina L, Culubret M, Bosch FX and de Sanjosé S: Underscreened women remain overrepresented in the pool of cervical cancer cases in spain: A need to rethink the screening interventions. Biomed Res Int 2015: 605375, 2015.

7. Safaeian M, Solomon D and Castle PE: Cervical cancer prevention-cervical screening: Science in evolution. Obstet Gynecol Clin North Am 34: 739-760, ix, 2007.

8. Karimi-Zarchi M, Peighmbari F, Karimi N, Rohi M and Chiti Z: A Comparison of 3 Ways of Conventional Pap Smear, Liquid-Based Cytology and Colposcopy vs Cervical Biopsy for Early Diagnosis of Premalignant Lesions or Cervical Cancer in Women with Abnormal Conventional Pap Test. Int J Biomed Sci 9: 205-210, 2013.

9. Franco EL: Chapter 13: Primary screening of cervical cancer with human papillomavirus tests. J Natl Cancer Inst Monogr 89-96, 2003.

10. Kulasingam SL, Havrilesky LJ, Ghebre R and Myers ER: Screening for cervical cancer: A modeling study for the US preventive services task force. J Low Genit Tract Dis 17: 193-202, 2013.

11. Marlow LA, Waller J and Wardle J: Barriers to cervical cancer screening among ethnic minority women: A qualitative study. J Fam Plann Reprod Health Care 41: 248-254, 2015.

12. McLaughlin-Drubin ME and Munger K: Viruses associated with human cancer. Biochim Biophys Acta 1782: 127-150, 2008.

13. Parkin DM: The global health burden of infection-associated cancers in the year 2002. Int J Cancer 118: 3030-3044, 2006.

14. Combes JD, Pawlita M, Waterboer T, Hammouda D, Rajkumar T, Vanhems P, Snijders P, Herrero R, Franceschi S and Clifford G: Antibodies against high-risk human papillomavirus proteins as markers for invasive cervical cancer. Int J Cancer 135: 2453-2461, 2014.

15. Coghill AE and Hildesheim A: Epstein-Barr virus antibodies and the risk of associated malignancies: Review of the literature. Am J Epidemiol 180: 687-695, 2014.

16. Mahieux R and Gessain A: Adult T-cell leukemia/lymphoma and HTLV-1. Curr Hematol Malig Rep 2: 257-264, 2007.

17. Walboomers JM,Jacobs MV, Manos MM, Bosch FX, Kummer JA, Shah KV, Snijders PJ, Peto J, Meijer CJ and Muñoz N: Human papillomavirus is a necessary cause of invasive cervical cancer worldwide. J Pathol 189: 12-19, 1999.

18. Luevano M, Bernard HU, Barrera-Saldaña HA, Trevino V, Garcia-Carranca A, Villa LL, Monk BJ, Tan X, Davies DH, Felgner PL and Kalantari M: High-throughput profiling of the humoral immune responses against thirteen human papillomavirus types by proteome microarrays. Virology 405: 31-40, 2010.

19. Joura EA, Ault KA, Bosch FX, Brown D, Cuzick J, Ferris D, Garland SM, Giuliano AR, Hernandez-Avila M, Huh W, et al: Attribution of 12 high-risk human papillomavirus genotypes to infection and cervical disease. Cancer Epidemiol Biomarkers Prev 23: 1997-2008, 2014.

20. Burd EM: Human papillomavirus and cervical cancer. Clin Microbiol Rev 16: 1-17, 2003.

21. Lutz HU: Homeostatic roles of naturally occurring antibodies: An overview. J Autoimmun 29: 287-294, 2007.

22. Schwartz-Albiez R, Monteiro RC, Rodriguez M, Binder CJ and Shoenfeld Y: Natural antibodies, intravenous immunoglobulin and their role in autoimmunity, cancer and inflammation. Clin Exp Immunol 158 (Suppl 1): S43-S50, 2009.

23. Peng Y, Kowalewski R, Kim S and Elkon KB: The role of IgM antibodies in the recognition and clearance of apoptotic cells. Mol Immunol 42: 781-787, 2005. 
24. Desmetz C, Mange A, Maudelonde T and Solassol J: Autoantibody signatures: Progress and perspectives for early cancer detection. J Cell Mol Med 15: 2013-2024, 2011.

25. Knight V, Merkel PA and O'Sullivan MD: Anticytokine autoantibodies: Association with infection and immune dysregulation. Antibodies 5, 2016. doi: 10.3390/antib5010003.

26. Wu J and Li L: Autoantibodies in Alzheimer's disease: Potential biomarkers, pathogenic roles, and therapeutic implications. J Biomed Res 30: 361-372, 2016.

27. Arbuckle MR, McClain MT, Rubertone MV, Scofield RH, Dennis GJ, James JA and Harley JB: Development of autoantibodies before the clinical onset of systemic lupus erythematosus. N Engl J Med 349: 1526-1533, 2003.

28. Lacombe J, Mange A and Solassol J: Use of Autoantibodies to Detect the Onset of Breast Cancer. J Immunol Res 2014: 574981 , 2014.

29. Liu Y, Liao Y, Xiang L, Jiang K, Li S, Huangfu M and Sun S: A panel of autoantibodies as potential early diagnostic serum biomarkers in patients with breast cancer. Int J Clin Oncol 22: 291-296, 2017

30. Huangfu M, Xu S, Li S, Sun B, Lee KH, Liu L and Sun S: A panel of autoantibodies as potential early diagnostic serum biomarkers in patients with cervical cancer. Tumour Biol 37: 8709-8714, 2016.

31. Tan EM and Zhang J: Autoantibodies to tumor-associated antigens: Reporters from the immune system. Immunol Rev 222: 328-340, 2008

32. Zhang JY, Casiano CA, Peng XX, Koziol JA, Chan EK and Tan EM: Enhancement of antibody detection in cancer using panel of recombinant tumor-associated antigens. Cancer Epidemiol Biomarkers Prev 12: 136-143, 2003.

33. Tabuchi Y, Shimoda M, Kagara N, Naoi Y, Tanei T, Shimomura A Shimazu K, Kim SJ and Noguchi S: Protective effect of naturally occurring anti-HER2 autoantibodies on breast cancer. Breast Cancer Res Treat 157: 55-63, 2016.

34. Shih NY, Lai HL, Chang GC, Lin HC, Wu YC, Liu JM, Liu KJ and Tseng SW: Anti-alpha-enolase autoantibodies are down-regulated in advanced cancer patients. Jpn J Clin Oncol 40: 663-669, 2010

35. Van Hoesen K, Meynier S, Ribaux P, Petignat P, Delie F and Cohen M: Circulating GRP78 antibodies from ovarian cancer patients: A promising tool for cancer cell targeting drug delivery system? Oncotarget 8: 107176-107187, 2017.

36. Pinho SS and Reis CA: Glycosylation in cancer: Mechanisms and clinical implications. Nat Rev Cancer 15: 540-555, 2015.

37. Krueger KE and Srivastava S: Posttranslational protein modifications: Current implications for cancer detection, prevention and therapeutics. Mol Cell Proteomics 5: 1799-1810, 2006.

38. He Y, Zhou Z, Hofstetter WL, Zhou Y, Hu W, Guo C, Wang L, Guo W, Pataer A, Correa AM, et al: Aberrant expression of proteins involved in signal transduction and DNA repair pathways in lung cancer and their association with clinical parameters. PLoS One 7: e31087, 2012.

39. Geradts J and Ingram CD: Abnormal expression of cell cycle regulatory proteins in ductal and lobular carcinomas of the breast. Mod Pathol 13: 945-953, 2000.

40. Zaenker P, Gray ES and Ziman MR: Autoantibody Production in Cancer-The Humoral Immune Response toward Autologous Antigens in Cancer Patients. Autoimmun Rev 15: 477-483, 2016.

41. Pappa KI, Lygirou V, Kontostathi G, Zoidakis J, Makridakis M, Vougas K, Daskalakis G, Polyzos A and Anagnou NP: Proteomic analysis of normal and cancer cervical cell lines reveals deregulation of cytoskeleton-associated proteins. Cancer Genomics Proteomics 14: 253-266, 2017.

42. Kontostathi G, Zoidakis J, Makridakis M, Lygirou V, Mermelekas G, Papadopoulos T, Vougas K, Vlamis-Gardikas A, Drakakis P, Loutradis D, et al: Cervical cancer cell line secretome highlights the roles of transforming growth factor-Beta-induced protein ig-h3, peroxiredoxin-2, and NRF2 on cervical carcinogenesis. Biomed Res Int 2017: 4180703, 2017.

43. Demarco M, Lorey TS, Fetterman B, Cheung LC, Guido RS Wentzensen N, Kinney WK, Poitras NE, Befano B, Castle PE, et al: Risks of CIN 2+, CIN 3+, and cancer by cytology and human papillomavirus status: The foundation of risk-based cervical screening guidelines. J Low Genit Tract Dis 21: 261-267, 2017.

44. Holowaty P, Miller AB, Rohan T and To T: Natural history of dysplasia of the uterine cervix. J Natl Cancer Inst 91: 252-258, 1999.

45. Ostör AG: Natural history of cervical intraepithelial neoplasia: A critical review. Int J Gynecol Pathol 12: 186-192, 1993.
46. McCredie MR, Sharples KJ, Paul C, Baranyai J, Medley G, Jones RW and Skegg DC: Natural history of cervical neoplasia and risk of invasive cancer in women with cervical intraepithelial neoplasia 3: A retrospective cohort study. Lancet Oncol 9: 425-434, 2008

47. Macdonald IK, Parsy-Kowalska CB and Chapman CJ: Autoantibodies: Opportunities for early cancer detection. Trends Cancer 3: 198-213,2017.

48. Zaenker P and Ziman MR: Serologic autoantibodies as diagnostic cancer biomarkers-a review. Cancer Epidemiol Biomarkers Prev 22: 2161-2181, 2013.

49. Pedersen JW and Wandall HH: Autoantibodies as Biomarkers in Cancer. Lab Medicine 42:623-628, 2011.

50. de Oliveira GA, Rangel LP, Costa DC and Silva JL: Misfolding, aggregation, and disordered segments in c-Abl and p53 in human cancer. Front Oncol 5: 97, 2015.

51. Shoenfeld Y and Toubi E: Protective autoantibodies: Role in homeostasis, clinical importance, and therapeutic potential. Arthritis Rheum 52: 2599-2606, 2005.

52. Siloşi I, Siloşi CA, Boldeanu MV, Cojocaru M, Biciuşcă V, Avrămescu CS, Cojocaru IM, Bogdan M and FolcuTi RM: The role of autoantibodies in health and disease. Rom J Morphol Embryol 57 (Suppl): 633-638, 2016.

53. Cohen $M$ and Petignat P: Purified autoantibodies against glucose-regulated protein 78 (GRP78) promote apoptosis and decrease invasiveness of ovarian cancer cells. Cancer Lett 309: 104-109, 2011.

54. Díaz-Zaragoza M, Hernández-Ávila R, Viedma-Rodríguez R, Arenas-Aranda D and Ostoa-Saloma P: Natural and adaptive IgM antibodies in the recognition of tumor-associated antigens of breast cancer (Review). Oncol Rep 34: 1106-1114, 2015.

55. Nagele EP, Han M, Acharya NK, DeMarshall C, Kosciuk MC and Nagele RG: Natural IgG autoantibodies are abundant and ubiquitous in human sera, and their number is influenced by age, gender, and disease. PLoS One 8: e60726, 2013.

56. Krasnov GS, Dmitriev AA, Snezhkina AV and Kudryavtseva AV: Deregulation of glycolysis in cancer: Glyceraldehyde-3-phosphate dehydrogenase as a therapeutic target. Expert Opin Ther Targets 17: 681-693, 2013

57. Hansen CN, Ketabi Z, Rosenstierne MW, Palle C, Boesen HC and Norrild B: Expression of CPEB, GAPDH and U6snRNA in cervical and ovarian tissue during cancer development. APMIS 117: 53-59, 2009

58. Kim JW, Kim SJ, Han SM, Paik SY, Hur SY, Kim YW, Lee JM and Namkoong SE: Increased glyceraldehyde-3-phosphate dehydrogenase gene expression in human cervical cancers. Gynecol Oncol 71: 266-269, 1998.

59. Zheng J: Energy metabolism of cancer: Glycolysis versus oxidative phosphorylation (Review). Oncol Lett 4: 1151-1157, 2012.

60. Altenberg B and Greulich KO: Genes of glycolysis are ubiquitously overexpressed in 24 cancer classes. Genomics 84 : 1014-1020, 2004

61. Liu K, Tang Z, Huang A, Chen P, Liu P, Yang J, Lu W, Liao J, Sun Y, Wen S, et al: Glyceraldehyde-3-phosphate dehydrogenase promotes cancer growth and metastasis through upregulation of SNAIL expression. Int J Oncol 50: 252-262, 2017.

62. Hao L, Zhou X, Liu S, Sun M, Song Y, Du S, Sun B, Guo C, Gong L, Hu J, et al: Elevated GAPDH expression is associated with the proliferation and invasion of lung and esophageal squamous cell carcinomas. Proteomics 15: 3087-3100, 2015.

63. Nicholls C, Pinto AR, Li H, Li L, Wang LH, Simpson R and Liu JP: Glyceraldehyde-3-phosphate dehydrogenase (GAPDH) induces cancer cell senescence by interacting with telomerase RNA component. Proc Natl Acad Sci USA 109:13308-13313, 2012.

64. Jung DW, Kim WH, Seo S, Oh E, Yim SH, Ha HH, Chang YT and Williams DR: Chemical targeting of GAPDH moonlighting function in cancer cells reveals its role in tubulin regulation. Chem Biol 21: 1533-1545, 2014.

65. Lea MA, Qureshi MS, Buxhoeveden M, Gengel N, Kleinschmit J and Desbordes C: Regulation of the proliferation of colon cancer cells by compounds that affect glycolysis, including 3-bromopyruvate, 2-deoxyglucose and biguanides. Anticancer Res 33: 401-407, 2013.

66. Kim JW, Kim TE, Kim YK, Kim YW, Kim SJ, Lee JM, Kim IK and Namkoong SE: Antisense oligodeoxynucleotide of glyceraldehyde-3-phosphate dehydrogenase gene inhibits cell proliferation and induces apoptosis in human cervical carcinoma cell lines. Antisense Nucleic Acid Drug Dev 9: 507-513, 1999. 
67. Chapman CJ,Healey GF, Murray A,Boyle P, Robertson C, Peek LJ, Allen J, Thorpe AJ, Hamilton-Fairley G, Parsy-Kowalska CB, et al EarlyCDT ${ }^{\circledR}$-Lung test: Improved clinical utility through additional autoantibody assays. Tumour Biol 33: 1319-1326, 2012.

68. Jin Y, Kim SC, Kim HJ, Ju W, Kim YH and Kim HJ: Use of autoantibodies against tumor-associated antigens as serum biomarkers for primary screening of cervical cancer. Oncotarget 8: 105425-105439, 2017.

69. Révillion F, Pawlowski V, Hornez L and Peyrat JP: Glyceraldehyde-3-phosphate dehydrogenase gene expression in human breast cancer. Eur J Cancer 36: 1038-1042, 2000.
70. Tokunaga K, Nakamura Y, Sakata K, Fujimori K, Ohkubo M, Sawada K and Sakiyama S: Enhanced expression of a glyceraldehyde-3-phosphate dehydrogenase gene in human lung cancers. Cancer Res 47: 5616-5619, 1987.

71. Hjerpe E, Egyhazi Brage S, Carlson J, Frostvik Stolt M, Schedvins K, Johansson H, Shoshan M and Avall-Lundqvist E: Metabolic markers GAPDH, PKM2, ATP5B and BEC-index in advanced serous ovarian cancer. BMC Clin Pathol 13: 30, 2013.

72. Abdel-Haleem AM, Lewis NE, Jamshidi N, Mineta K, Gao X and Gojobori T: The emerging facets of non-cancerous warburg effect. Front Endocrinol (Lausanne) 8: 279, 2017. 